\title{
DEVELOPING SYSTEMS VISUALISATIONS IN DESIGN THROUGH A TYPOLOGY OF VISUAL TASKS: A MECHATRONIC CASE
}

\author{
Idrissov, Agzam (1); \\ Rapp, Simon (2); \\ Albers, Albert (2); \\ Maier, Anja M. (1) \\ 1: DTU - Technical University of Denmark; \\ 2: KIT - Karlsruhe Institute of Technology
}

\begin{abstract}
Visual representations are essential to design. Data-rich representations such as systems visualisations are gaining prominence in engineering practice. However, as such visualisations are often developed ad-hoc, we propose more systematically to link visual tasks with design-specific tasks for which the visualisations are used. Whereas research on such linking focuses mostly on CAD models and sketches, no such studies are yet available for systems visualisations. Thus, this paper introduces a typology of visual tasks from the Information Visualisation field to aid the development of systems visualisations in design. To build a visualisation using the typology, a case study with engineering students developing an autonomous robot was conducted. Through interviews and analysis of product representations used, design-specific tasks were identified and decomposed into visual tasks. Then, a visualisation that assisted the team in performing their design activities was created. Results illustrate the benefits of using such a typology to describe visual tasks and generate systems visualisations. The study suggests implications for researchers studying visual representations in design as well as for developers of systems visualisations.
\end{abstract}

Keywords: Visualisation, Design process, Mechatronics, Visual representation, Design activity

\section{Contact:}

Idrissov, Agzam

DTU - Technical University of Denmark

Engineering Systems Group

Denmark

agzid@dtu.dk 


\section{INTRODUCTION}

Visual representations are central in engineering design (Henderson, 1998). As the complexity of products grows and the digitalisation of design processes sees a shift from document-centric to a model-centric view on design (Albers et al., 2019; Huldt and Stenius, 2019), designers are even more required to make sense of large amounts of highly interconnected and dynamic engineering data; data that cuts across multiple design domains (e.g. on requirements, on the overall systems architecture, on verification tests) and multiple engineering disciplines (e.g. mechanical or software). For that, computer-supported, interactive, and data-rich visual representations, referred to as systems visualisations, are increasingly used in practice. Such visualisations are capable of displaying large amounts of data, highlighting trends, errors and outliers within the data, or aiding navigation between different levels of granularity of data (Shneiderman, 2003). However, despite these capabilities to represent engineering data, the use of systems visualisations currently worked with in practice is challenging (Hopf and Ovtcharova, 2016). This often appears due to mismatches between the visual tasks (such as searching) that a designer or other stakeholders perform when engaging with such visualisations and the encoding used in a given visualisation (such as aligning) and interactive techniques (such as filtering) that would be most appropriate for a given design task. As such, we propose to incorporate visual tasks into developing systems visualisations. Such studies have been performed for other types of representations, including sketches (Suwa and Tversky, 1997) and CAD models (Bilda and Demirkan, 2003). Although current research on design activities provides insights into designers' actions at multiple levels of granularity (Cash and Kreye, 2017; Hubka and Eder, 1988; Sim and Duffy, 2003), more detailed knowledge on the working principles of visualisations is required. Drawing from the field of Information Visualisation, this paper proposes to use visual tasks (Amar et al., 2005; Wehrend and Lewis, 1990), often used interchangeably with visualisation tasks, also referred to as "abstract tasks" by (Brehmer and Munzner, 2013) and defined as "domain and interface-agnostic operations performed by users" (Munzner, 2014). When designers as users of visualisations engage with visualisations, they perform visual tasks. A design-specific task may be to "search for optimal product configuration arrangements of components" and examples of visual tasks might then include "finding if connections between two nodes in a graph exist" or "understanding the hierarchical structure of a tree". The proposition of this paper is that by translating design-specific tasks into visual tasks, the selection of appropriate visualisation techniques can then be addressed explicitly and inform the building of visualisations. The aim of the paper is to explore this mapping of design-specific tasks and visual tasks in a practice case.

The remainder of the paper is structured as follows. First, a literature background introduces state-ofthe-art in systems visualisations in the field of Design and a typology of visual tasks from the field of Information Visualisations, with the proposition to link design-specific tasks to visual tasks as a prerequisite for developing more appropriate systems visualisations. Third, the case of designing an autonomous beverage delivery robot that involves building a visualisation using the typology is described. Finally, the results obtained are discussed in connection with relevant literature. Implications for research and practice conclude the paper.

\section{LITERATURE BACKGROUND}

\subsection{Systems visualisations in design}

A range of systems visualisations is being proposed to support design activities. For instance, an annotated collapsible hierarchical tree was introduced by Bracewell et al. (2009) and deployed, e.g. at Rolls-Royce plc, to capture design rationale. Using fish-eye network visualisations, Keller et al. (2005) study and assess change propagation, e.g. during helicopter design. Fleming et al. (2005) utilise parallel coordinates plot (Inselberg, 1985) to perform multi-criteria optimisation during the design of a flight control system. However, such studies do not report using a structured approach to describe the visual tasks that are involved. If visualisations are created solely based on design-specific tasks without a close understanding of what visual tasks are performed by designers, this may lead to choosing suboptimal visualisations, which limits design space exploration (Chandrasegaran et al., 2013). Studies have shown that visualisation options embedded in commercially available software and widely used by manufacturing firms have several deficiencies (Gebhardt et al., 2014; Gotel et al., 2007; Guo et al., 2014). These deficiencies, such as an inappropriate selection of visualisations 
embedded in the software or sub-optimal types or layouts of visual elements, prevent designers from performing visual tasks such as finding links between nodes of a graph or analysing the hierarchical structure of a tree diagram in an efficient manner (i.e. quickly and using minimal cognitive work (Ware, 2012)). Moreover, without a structured approach to analyse visual tasks in design, it becomes non-trivial to compare and evaluate visualisations in their capability to support specific design tasks.

In the design context, research on visual tasks for systems visualisations is limited compared to other visual representations. For instance, Suwa and Tversky (1997) examine dependencies between design activities and information content of design problems (such as emergent properties, spatial and functional relations, background knowledge) for sketching. Bilda and Demirkan (2003) inspect the goals and sub-goals of designers working with sketches and CAD models, including visual tasks. Research on working principles of systems visualisations is needed as they show differences compared to other visual representations in design, e.g. they are less detailed than CAD models and are more structurally restrictive than sketches (Bresciani, 2019).

Several papers attempt to categorise visualisations in design. For instance, Bresciani (2019) proposes to classify visualisations according to characteristics such as Content Modifiability, Structural Restrictiveness, Outcome Clarity, Perceived Finishedness and Collaboration Support. While taxonomies of this kind help choose appropriate visualisation types, a more elaborate understanding of other visualisation aspects is required, such as encoding of visual elements, for example, through colour, shape, or size and interaction techniques, for example, navigation, aggregation, or filtering (Brehmer and Munzner, 2013; Munzner, 2014). For instance, even if the visualisation type is selected in accordance with the visual tasks, for example, an adjacency matrix is chosen to visualise connections between entities, crucial interactive features, such as filtering and sorting, might be missing, which limits designers' capability to manipulate the visualisation and generate better designs. Finally, several studies focus on classifying design activities. For example, Sim and Duffy (2003) categorise design activities into a definition, management, and evaluation and link them to goals that drive said activities and to the respective knowledge required. Cash et al. (2019) extend this work by focusing on more granular operations that they term design actions. Design actions are organised into information-, knowledge-sharing-, and representation actions. Although these works greatly advance our understanding of the nature of design activities, they do not explicitly focus on visual tasks, which makes them hard directly to apply during the development of systems visualisations.

These literature gaps point to the need for understanding the interrelationships between visualisations and visual tasks within the context of design activities. Therefore, in the next section, we draw on seminal works from the field of Information Visualisation, which provides a vocabulary to translate design-specific tasks into visual tasks and linking the two.

\subsection{A typology of visual tasks}

Systems visualisations are particularly focused on within the research field of Information Visualisation, which provides a body of theory and empirical evidence to increase the efficiency of systems visualisations in representing data. For instance, multiple taxonomies are proposed to categorise main aspects of visualisations, such as input data types (Shneiderman, 2003) or utilised encoding and interaction techniques (Beck et al., 2017; Ellis and Dix, 2007; Keim, 2002).

Tailoring visualisations to support design activities requires modelling of interrelationships between users' actions and what the visualisations allow the users to accomplish, i.e. the visualisations' functions. While there exist several frameworks that classify visual tasks (Amar et al., 2005; Wehrend and Lewis, 1990), a typology by Brehmer and Munzner (2013) and refined by Munzner (2014) synthesises several existing typologies, overarchingly to describe visualisation tasks (often interchangeably used with visual tasks) and the functions of visualisations on multiple levels of granularity. Brehmer and Munzner (2013) and Munzner (2014) refer to visualisation tasks as "abstract tasks" and defined as "domain and interface-agnostic operations performed by users" when engaging with visualisations. From here forward, we use the term "visual tasks".

Brehmer and Munzner's (Brehmer and Munzner, 2013; Munzner, 2014) typology of visual tasks comprises three parts that answer questions about visualisations: what is being visualised, why a user engages with the visualisation and how does the visualisation address user needs. Clarifying all three parts of the typology guides decisions about visualisation techniques. While a detailed description of the typology is discussed elsewhere (Brehmer and Munzner, 2013; Munzner, 2014), in what follows, three parts of the typology are reviewed, as shown in Table 1, and relevant examples are provided. 
Table 1. A typology of visual tasks

(re-created from (Brehmer and Munzner, 2013; Munzner, 2014))

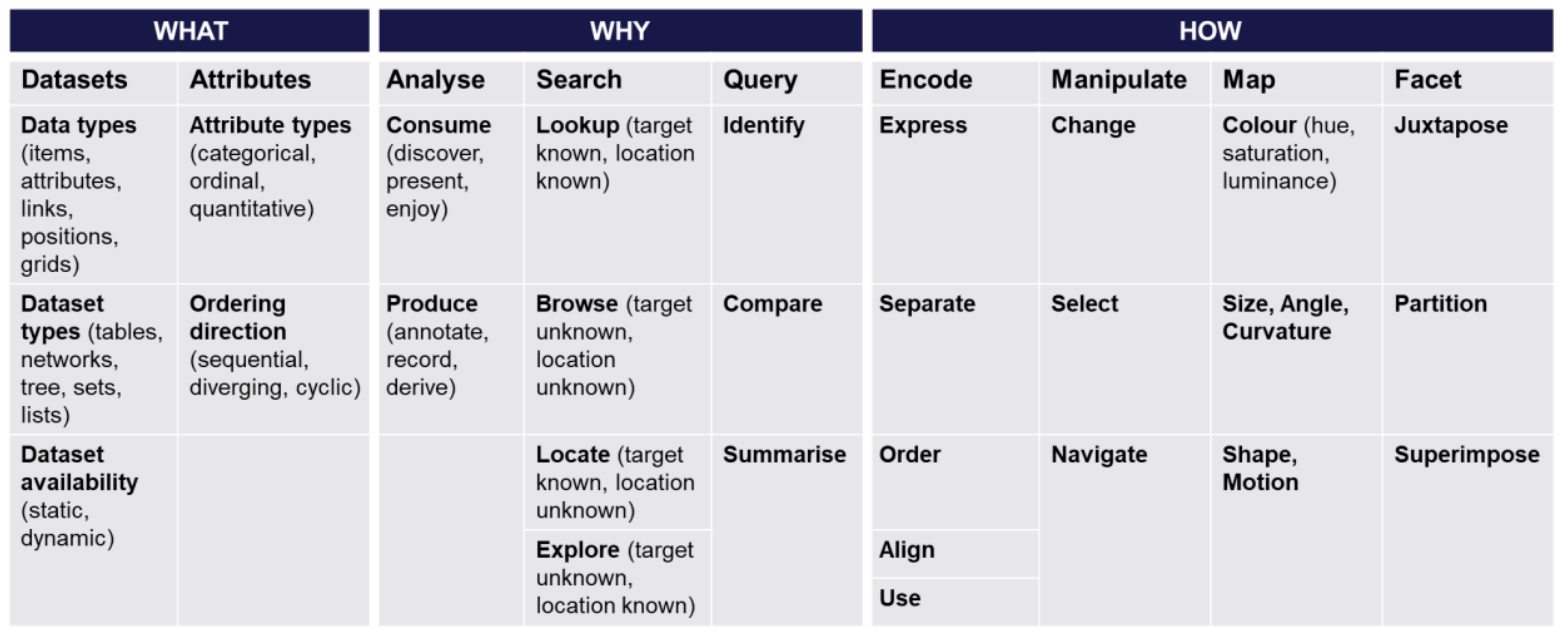

- The What of the typology analyses what kind of data needs to be visualised. Data may include items, their attributes and links between items (Munzner, 2014). Such data may be structured in the form of a table, network, field (e.g. a grid with a continuous range of positions) and a geometry (e.g. spatial description of 2D curves) (Munzner, 2014). Moreover, attributes themselves could be categorical or ordered with their own direction of order. Although understanding the structure of data is equally crucial to the choice of the employed visualisation type, in this paper, the authors specifically focus on visual tasks, i.e. on connections between the Why and How part of the typology, while the What part is well covered elsewhere (e.g. Larkin et al., 1987).

- The Why describes the purpose with which visualisation is used on multiple levels. At the very top level, a user engages with the visualisation to analyse the information. Then, the information can be either consumed or produced through the visualisation. When consuming the information, potential tasks could be to discover new knowledge from the given information or to present the information to others. The original typology includes the third task - to enjoy the visualisation, although the authors could not find evidence of such use in the design literature. When new information is produced through the visualisation, a user can either annotate the visualisation by adding new information onto the visualisation by manipulating it, record the state of the visualisation in a different format (e.g. a database or a screenshot) or derive new attributes of data from the existing ones (e.g. transform quantitative sensor readings into qualitative high, medium, low values) (Munzner, 2014).

At the next level, a user performs different types of information search tasks depending on whether the search target and location are known. If the user knows what to search for and where, the task is to lookup that information. If the target is known, but the location is not known, a user performs a search by locating the target. In contrast, if the target is unknown but the location is known, the user browses the visualisation. When both target and location are not known, a user overviews the information by exploring the visualisation (Munzner, 2014).

Finally, the bottom level of tasks describes the mode of querying the data. Depending on the number of targets, a task could be to identify one target, compare two or more targets or summarise all targets altogether (Munzner, 2014).

- The How of the typology describes an assortment of functions of the visualisation and is broken down into encoding, manipulation, faceting and reducing techniques. Encoding techniques determine the spatial layout or type of the visual elements used (e.g. matrix or network diagram). Manipulation techniques include changing the positions or shapes of visual elements, selecting elements of interest or navigating around the visualisation (e.g. scrolling or zooming in or out). Faceting techniques use multiple coordinated views (Baldonado et al., 2000) or small multiples (Scherr, 2008) techniques to show differences or similarities between the views. Finally, reducing techniques include filtering out unnecessary information, aggregating visual elements to alter granularity of information or embedding additional explanations of the data (e.g. displaying a tooltip when hovering a cursor over an element of interest) (Munzner, 2014).

The typology aims to inform the development of visualisations ultimately to support design activities. After the structure of engineering data (e.g. existing tables or diagrams) is determined, design-specific 
tasks within design activities are translated into visual tasks. An example of a design-specific task could be "to understand functional decomposition of a product", which is translated into a visual task "to explore the hierarchy of entities". Once visual tasks are established, the developer of the visualisation proceeds to select appropriate visualisation techniques, focusing on the How part of the typology.

The next section describes a case from mechatronic design of an autonomous beverage delivery robot, where the typology was used to inspect visual tasks and assist the development of a visualisation in supporting system-based design activities.

\section{CASE: USING THE TYPOLOGY TO BUILD THE VISUALISATION}

To understand the usefulness of the typology to analyse visual tasks and to inform building the visualisations, the authors followed the process of designing an autonomous robot and developed a visualisation aimed to support the respective design activities. The authors' goal was to examine whether building a visualisation based on the identified visual tasks and using visualisation techniques to address these tasks would support the study participants in their design activities.

The study was performed during a graduate product development course that runs annually over ten months at a technical university in Germany. Course participants had to design the second generation of an autonomous beverage delivery robot, the first generation of which was developed by the previous course intake a year before. The design team consisted of six graduate students working parttime in automotive and aerospace industries and were given and grouped into the following roles: two product managers, a systems engineer, a software engineer, a structural engineer and a CAD expert.

The visualisation was constructed dynamically throughout the design process by iteratively incorporating feedback from the design participants and changes in the documentation. The development of the visualisation was performed in three iterations, where one iteration cycle included a demonstration of the visualisation, followed by a feedback session from the participants and incorporating the feedback results into the new version of the visualisation.

\subsection{Building the visualisation}

\subsubsection{Understanding the current data and visual representations}

At the start of the study, the design team provided access to their current working shared folder that served as a document repository of engineering data and included the representations illustrated in Figure 1. In essence, the team modelled the product using four design domains: requirements, use cases, architecture and stakeholders. The linkages between these design domains were represented using common visual representations: entity-relationship diagrams, tables and lists.

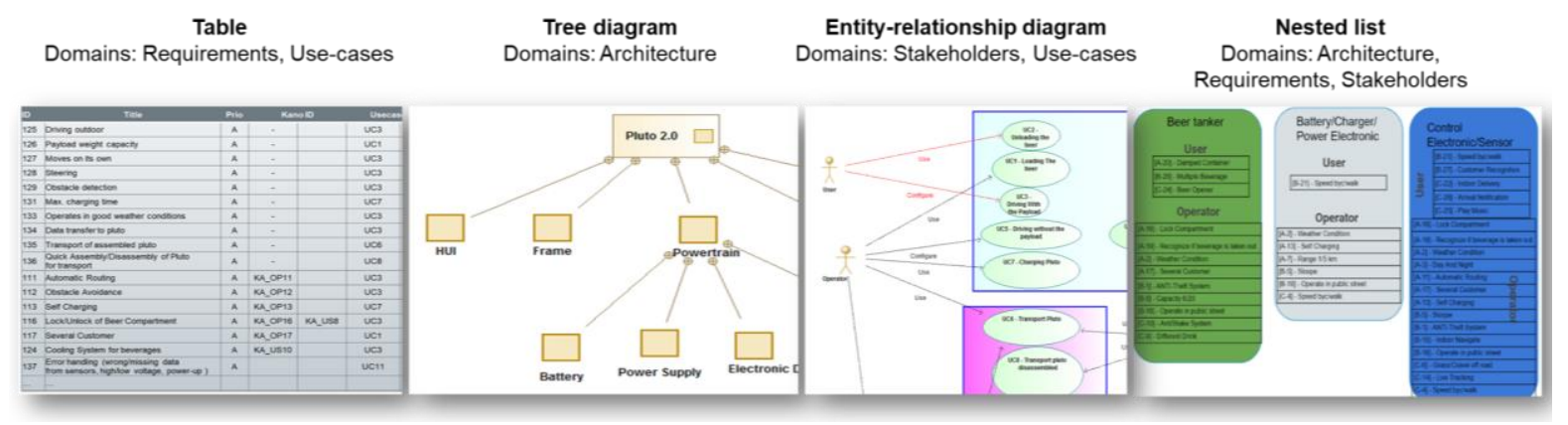

Figure 1. Visual representations of engineering data prior to the studied case

\subsubsection{Using the typology of visual tasks to support the creation of an integrated visualisation}

First, the authors have identified what type of data the team used, clarifying the What part of the typology. As seen in Figure 1, data and representations used by the team prior to the study to represent design domains and their connections mostly resembled graph and tree structures. Once the structure of the data was examined, a number of visual tasks that needed to be supported by the visualisation were derived, addressing the why part of the typology. Table 2 presents the overview obtained of design-specific-, generic analytical (non-design-specific)-, and visual tasks. For instance, a common 
design-specific task, such as to understand current product architecture (further referred to as Task A), translates into the generic analytical task to overview the hierarchical structure and further into the following sequence of visual tasks: Analyse:Discover - Search:Explore - Query:Summarise.

Table 2. The typology of visual tasks for design activities used in the case studied

\begin{tabular}{|c|c|c|c|}
\hline & Elicited during case interviews & Derived from design-specific tasks & $\begin{array}{l}\text { Derived from generic analytical tasks based on } \\
\text { Brehmer and Munzner (2013), Munzner (2014) }\end{array}$ \\
\hline Task name & Design-specific tasks & Generic analytical tasks & Visual tasks \\
\hline Task A & $\begin{array}{l}\text { to understand the current } \\
\text { product architecture }\end{array}$ & $\begin{array}{l}\text { to overview the hierarchical } \\
\text { structure }\end{array}$ & $\begin{array}{l}\text { Analyse:Consume:Discover } \\
\text { Search:Explore } \\
\text { Query:Summarise }\end{array}$ \\
\hline \multirow[t]{2}{*}{ Task B } & \multirow[t]{2}{*}{$\begin{array}{l}\text { to find requirements that } \\
\text { are covered by } \\
\text { architecture entities }\end{array}$} & \multirow[t]{2}{*}{$\begin{array}{l}\text { to discover the connections } \\
\text { between nodes of one } \\
\text { hierarchical tree and nodes of } \\
\text { another hierarchical tree }\end{array}$} & $\begin{array}{l}\text { Visual task 1: } \\
\text { Analyse:Consume:Discover } \\
\text { Search:Locate } \\
\text { Query:Identify }\end{array}$ \\
\hline & & & $\begin{array}{l}\text { Visual task 2: } \\
\text { Analyse:Consume:Discover } \\
\text { Search:Browse } \\
\text { Query:Identify }\end{array}$ \\
\hline Task C & $\begin{array}{l}\text { to document changes in } \\
\text { product information }\end{array}$ & to save updated data & $\begin{array}{l}\text { Analyse:Produce:Record } \\
\text { Search:Locate } \\
\text { Query:Identify }\end{array}$ \\
\hline
\end{tabular}

Another design-specific task, to find requirements that are covered by architecture entities (further referred to as Task B), was translated into a generic analytical task to discover connections between nodes of one hierarchical tree and nodes of another hierarchical tree. When users check connections to each requirement one-by-one, on the next, search and query levels, the task becomes to locate and identify a single connection repeatedly, accordingly. When such connections are being searched in an overview mode, e.g. by looking at a specific subtree, the task becomes to browse areas of the visualisation to identify several connections at once.

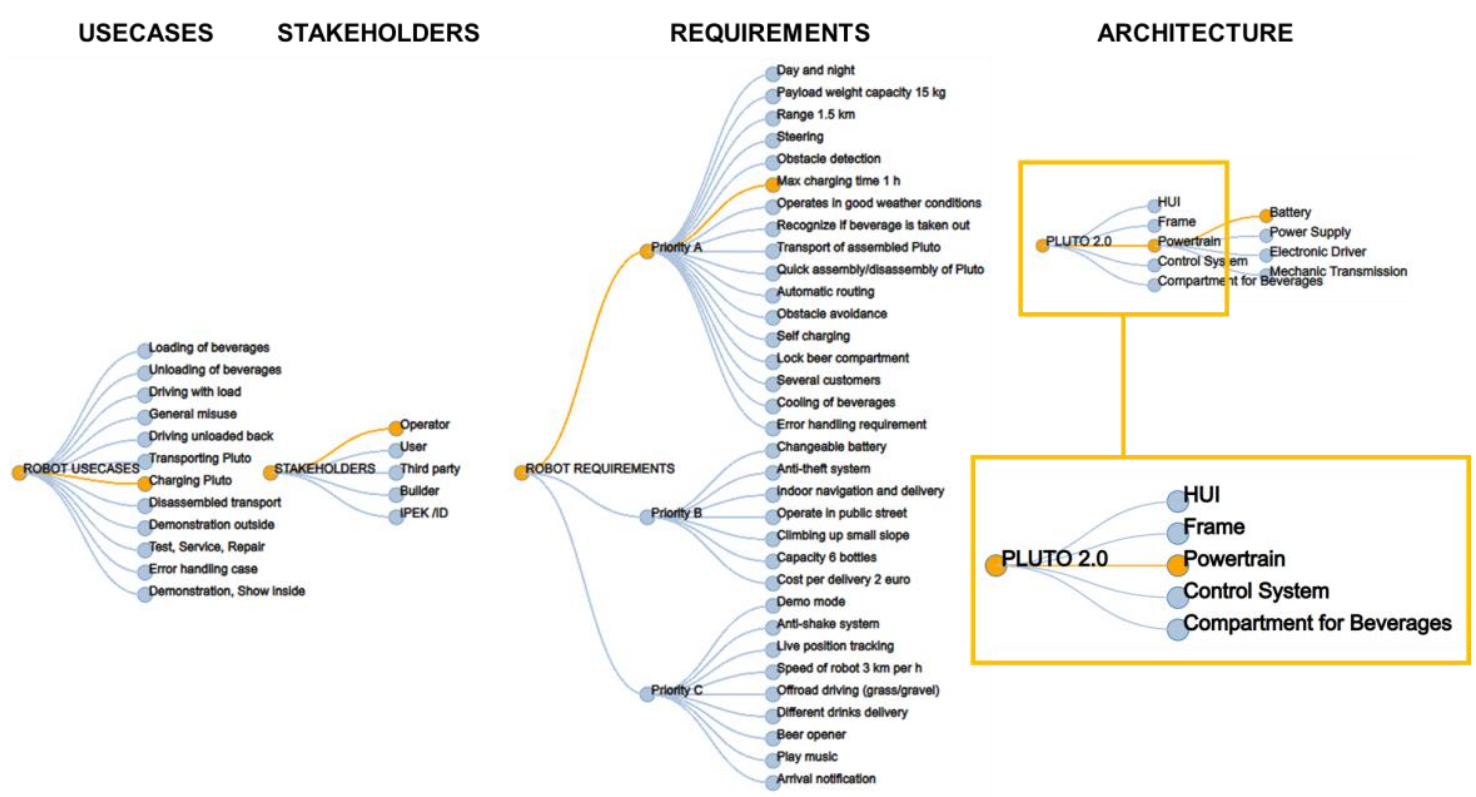

Figure 2. The developed visualisation of engineering data in the mechatronic case studied

Informed by the visual task analysis, the authors proceeded to dissect the How part of the typology by exploring the appropriate options of encoding and interaction techniques. Because of the tree structure of the data and related to task A, encoding the data via the hierarchical tree was fitting. Moreover, to account for cases when the number of nodes and levels of hierarchy becomes large, node collapsing and decollapsing (select + change) and zooming in and out (navigate) functionalities were added (Plaisant et al., 2002; Shneiderman, 2003). However, both tasks B and C require that connections 
across both requirement and architecture trees are visualised. Simply adding edges between the nodes would severely decrease the readability of the visualisation due to the edge crossing problem (Maier et al., 2014; Reingold and Fruchterman, 1991). Therefore, highlighting (Liang and Huang, 2010) connected nodes and edges (change) when hovering on a node of interest (select) was implemented. Employing these encoding and interaction techniques, a linked tree visualisation (Idrissov et al., 2019) was built, connecting four trees that represent each design domain, as shown in Figure 2.

\section{RESULTS}

The conducted feedback sessions drew attention to the performance of the developed systems visualisation on the outlined design-specific tasks. First, the visualisation provided the design participants with the means to trace connections across different design domains. Second, the developed visualisation enabled the overview of the designed system. Finally, the visualisation performed record-keeping functions and demonstrated utility to maintain documentation consistency.

\subsection{The visualisation built enabled a structured search process for new potential relationships between entities}

During the first feedback session, the team noted the benefits of the visualisation approach to trace connections between entities of different design domains. For instance, during the analysis of linkages between requirements to stakeholders and use cases, one of the team members noted:

"Especially for when you say: Ah, why is there a requirement for that and you can directly seeAh, there is this stakeholder linked to it, and there is this use case linked to it, so you will know directly why is this requirement there, not just for fun but has a clear connection to some stakeholder and also some use case, very needed actually". [product manager]

In another example, participants noticed that the lack of connection between a critical requirement that the robot should be able to be quickly assembled and disassembled for testing, maintenance and repair purposes.

"- Shouldn't requirement "Quick assembly/disassembly" also affect the use case "Test, Service and Repair"? [first product manager]

-Yeah, it would be actually right... Would make it definitely easier..." [systems engineer]

Thus, by representing how different design domains are connected, the visualisation enabled participants to take note of non-obvious links and missing elements, as required in design-specific task B.

\subsection{The visualisation built helped to overview the product architecture}

During the second feedback session, observation of requirements and architecture domains in the visualisation drew attention to the chosen way of organising subsystems within the architecture domain. In the instance described below, the team observed that the requirement "Max charging time 1 hour" is not linked to any component. This led to the discussion on how the battery charger should be implemented in relation to the other subsystems.

"Hmmm.. Charging time (requirement) is interesting because we haven't specified the charger". [systems engineer]

"We have a really rough idea of how it works, but we can start to speculate which systems are affected". [first product manager]

"But the battery is affected in the specification of it, for sure". [second product manager] In this example, the visualisation allowed the team to collectively think of acceptable ways to rearrange the robot's architecture, as intended by Task A and satisfying the outlined requirements.

\subsection{The visualisation built helped to maintain documentation consistency}

One additional and unexpected design-specific task emerged as the visualisation was presented to the designer team. During the second feedback session, one of the participants asked whether the visualisation automatically constructs the links between the domains that are not directly connected to each other. As the authors learned, the motivation behind this question was that the team "got an additional stakeholder requirement, so the design had to change", referring to an occasion when the supervising institute asked the team to demonstrate the performance of the robot both indoors and outdoors, which was not in the original requirements list for the second generation of the robot. Such adjustment in requirements led to the formulation of two new use cases, "Demonstration inside" and 
"Demonstration outside", one requirement "Demo mode" that addresses these use cases and one new stakeholder "Supervising Institute" and updating the connections within the model accordingly.

Regarding such a change, the team recalls modifying and maintaining the consistency of all the affected links in the system description in their PowerPoint and spreadsheet files as a rather time-consuming procedure that is prone to errors. One of the team members shared: "When everything is manual, you become miserable". While such use of the visualisation was not anticipated, the authors then translated this newly discovered task (Task C in Table 2) into a visual task of "Produce: Record - Search: LocateQuery: Identify". Then, based on this visual task, in the next development iteration of the built systems visualisation, the functionality "to add, remove, drag and drop nodes' and 'export the data to Microsoft Excel format" was added, although without subsequent evaluation by the participants.

\section{DIscussion}

The contributions of this paper are twofold. First, a typology of visual tasks from the field of Information Visualisation is presented to assist the development of visualisations during system-based design activities. Second, operationalising the typology in a design context, the authors built a visualisation to assist in designing of an autonomous beverage delivery robot. Using the typology, design-specific tasks of the design team were described as a set of visual tasks. Based on the identified set of visual tasks, an appropriate assortment of visual encoding and interaction techniques were selected and synthesised into one central visualisation. The generated visualisation demonstrated a capability to facilitate several design-specific tasks, such as the structured search of interconnections between entities, an overview of the overall product system architecture and maintenance of product documentation consistency.

This work has four central implications: First, the paper engages with the conversation on the use of visual representations in design by including systems visualisations and links design tasks and visual tasks. Suwa and Tversky (1997) analyse the information content of sketches which corresponds to the What part of the typology of visual tasks used and the design activity segments which correspond to the Why part of the typology by Brehmer and Munzner (2013) and Munzner (2014). Similarly, Bilda and Demirkan's decomposition of designers' goals and sub-goals when engaging with CAD models (2003) is parallel to the Why part. Thus, in a similar vein, another type of visual representation is discussed computer-supported, data-rich visualisations used in terms of visual tasks they help to accomplish (why), types of data they are suited to represent (what) and visual encoding and interaction techniques they have (how). Second, in line with existing design activity models (Cash and Kreye, 2017; Hubka and Eder, 1988; Sim and Duffy, 2003), the proposed typology of visual tasks makes interrelations between the visualised data and visual tasks explicit on a finer level of granularity than available in the current design literature. This allows a better understanding of the role of systems visualisations within design activities. Third, the paper further reinforces the works on profiling visualisations in design, specifically for systems visualisations. While Bresciani (2019) provides a comprehensive framework for choosing appropriate visual representation types (e.g. sketches, diagrams, CAD models) depending on the design activity, further fine-grained tuning of their functionality is often required to make them applicable in practice (Gotel et al., 2007; Guo et al., 2014). Fourth, the paper adds to applied literature on the approaches to develop visualisations to facilitate design activities. Compared to existing visualisation studies in design literature (overview provided in Konyha et al., 2009), this paper demonstrates that the choice for encoding and interaction techniques used in visualisations could be more closely related to the supported visual tasks during design activities. For instance, while a number of studies (Abi Akle et al., 2017; Keller et al., 2005) discuss the reasoning behind chosen visualisation techniques and mention separate examples of relevant visual tasks, presented typology of visual tasks provides a structured way of reasoning about the needs of designers and visualisation techniques to address these needs. This way, various visualisation concepts could be evaluated and compared with each other against the visual tasks they are tailored to aid.

In terms of practical relevance, as seen from the mechatronic case and indicated by Brehmer and Munzner (2013) and Munzner (2014), the typology can be used to describe visual tasks and assist the generation of visualisations. Given that the typology stems from the identification of specific visual tasks that need to be targeted, mismatches between the visualisation and the task it is designated to support are expected to be minimised. Moreover, different visual encoding and interaction techniques can be empirically evaluated and compared with respect to their usefulness for identified visual tasks. 
As the study participants used widespread software design support tools and followed a common product development methodology provided by the course instructors, the generated visual representations and design process can be said to include elements that resemble design practices in the industry. This suggests that observations presented here may be applicable beyond the setting described.

\section{CONCLUSION}

Visual representations are fundamental to the design process. As the complexity of the engineering data surrounding the design process grows, it is crucial to provide designers with appropriate visual representations that enable understanding of such data. The present study adds to the body of knowledge on the use of computer-supported, interactive, data-rich visualisations, namely systems visualisations. While design research has mostly focused on investigating the role of, e.g. sketches, diagrams, CAD models, or photographs, the current understanding of the working principles of systems visualisations is limited. Drawing on the field of Information Visualisation, the main contribution of the paper, therefore, has been to propose the use of a typology of visual tasks to inform the development of visualisations for design support tools. Visual tasks are mapped on to design tasks, and with that base, an improved visualisation is built. The case of the design and development of an autonomous robot is used as an example. The built interactive visualisation has shown that visual tasks could be described using the typology and that identified tasks were supported by the visualisation.

\section{ACKNOWLEDGEMENTS}

This research has partially been funded by the H2020 EU Framework Programme for Research and Innovation through the EURITO project under Grant Agreement $\mathrm{n}^{\circ} 770420$ and has also received financial support from the Karlsruhe House of Young Scientists (KHYS).

\section{REFERENCES}

Abi Akle, A., Yannou, B. and Minel, S. (2017), "Design space visualization for efficiency in knowledge discovery leading to an informed decision", Proceedings of the International Conference on Engineering Design, ICED, Vol. 6, pp. 061-070.

Albers, A., Bursac, N., Scherer, H., Birk, C., Powelske, J. and Muschik, S. (2019), "Model-based systems engineering in modular design", Design Science, Vol. 5, pp. 1-33, https://doi.org/10.1017/dsj.2019.15.

Amar, R., Eagan, J. and Stasko, J. (2005), "Low-level components of analytic activity in information visualization”, Proceedings - IEEE Symposium on Information Visualization, INFO VIS, pp. 111-117, https://doi.org/10.1109/INFVIS.2005.1532136.

Baldonado, M.Q.W., Woodruff, A. and Kuchinsky, A. (2000), "Guidelines for using multiple views in information visualization”, Workshop on Advanced Visual Interfaces, No. February 2013, pp. 110-119, https://doi.org/10.1145/345513.345271.

Beck, F., Burch, M., Diehl, S. and Weiskopf, D. (2017), “A Taxonomy and Survey of Dynamic Graph Visualization”, Computer Graphics Forum, Vol. 36 No. 1, pp. 133-159, https://doi.org/10.1111/cgf.12791.

Bilda, Z. and Demirkan, H. (2003), “An insight on designers' sketching activities in traditional versus digital media”, Design Studies, https://doi.org/10.1016/S0142-694X(02)00032-7.

Bracewell, R., Wallace, K., Moss, M. and Knott, D. (2009), “Capturing design rationale”, CAD Computer Aided Design, Elsevier, Vol. 41 No. 3, pp. 173-186, https://doi.org/10.1016/j.cad.2008.10.005.

Brehmer, M. and Munzner, T. (2013), “A multi-level typology of abstract visualization tasks”, IEEE Transactions on Visualization and Computer Graphics, Vol. 19 No. 12, pp. 2376-2385, https://doi.org/10.1109/TVCG.2013.124.

Bresciani, S. (2019), "Visual Design Thinking: A Collaborative Dimensions framework to profile visualisations", Design Studies, Elsevier Ltd, Vol. 63, pp. 92-124, https://doi.org/10.1016/j.destud.2019.04.001.

Cash, P. and Kreye, M. (2017), "Uncertainty Driven Action (UDA) model: A foundation for unifying perspectives on design activity", Design Science, Vol. 3, https://doi.org/10.1017/dsj.2017.28.

Cash, P., Škec, S. and Štorga, M. (2019), “The dynamics of design: exploring heterogeneity in meso-scale team processes”, Design Studies, Vol. 64, pp. 124-153, https://doi.org/10.1016/j.destud.2019.08.001.

Chandrasegaran, S.K., Ramani, K., Sriram, R.D., Horváth, I., Bernard, A., Harik, R.F. and Gao, W. (2013), “The evolution, challenges, and future of knowledge representation in product design systems", CAD Computer Aided Design, Vol. 45 No. 2, pp. 204-228, https://doi.org/10.1016/j.cad.2012.08.006. 
Ellis, G. and Dix, A. (2007), “A taxonomy of clutter reduction for information visualisation”, IEEE Transactions on Visualization and Computer Graphics, Vol. 13 No. 6, pp. 1216-1223, https://doi.org/10.1109/TVCG.2007.70535.

Fleming, P.J., Purshouse, R.C. and Lygoe, R.J. (2005), "Many-Objective Optimization: An Engineering Design Perspective", International Conference on Evolutionary Multi-Criterion Optimization, Springer, Berlin, pp. 14-32, https://doi.org/10.1007/978-3-540-31880-4_2.

Gebhardt, N., Beckmann, G. and Krause, D. (2014), "Visual representation for developing modular product families - Literature review and use in practice", DESIGN 2014, Vol. 2014-Janua, pp. 183-192.

Gotel, O.C.Z., Marchese, F.T. and Morris, S.J. (2007), “On requirements visualization”, 2nd International Workshop on Requirements Engineering Visualization, REV 2007, No. October 2007, https://doi.org/10.1109/REV.2007.4.

Guo, C., Chen, Y.V., Miller, C.L., Hartman, N.W., Mueller, A.B. and Connolly, P.E. (2014), "Information visualization for product lifecycle management (PLM) data”, ASEE Annual Conference and Exposition.

Henderson, K. (1998), “On Line and On Paper: Visual Representations, Visual Culture, and Computer Graphics in Design Engineering”, MIT Press Cambridge, MA, USA, p. 256.

Hopf, J.M. and Ovtcharova, J. (2016), "Deeper insights into product development through data visualization techniques", IFIP Advances in Information and Communication Technology, Vol. 467, pp. 485-494, https://doi.org/10.1007/978-3-319-33111-9_44.

Hubka, V. and Eder, W.E. (1988), Theory of Technical Systems, Theory of Technical Systems, https://doi.org/10.1007/978-3-642-52121-8.

Huldt, T. and Stenius, I. (2019), "State-of-practice survey of model-based systems engineering”, Systems Engineering, Vol. 22 No. 2, pp. 134-145, https://doi.org/10.1002/sys.21466.

Idrissov, A., Parraguez, P. and Maier, A.M. (2019), “Tracing Paths and Connecting Multiple Design Domains: An Information Visualisation Approach to Product Architecture Modelling", International Conference on Engineering Design (ICED’19), Vol. 1, pp. 3021-3030, https://doi.org/10.1017/dsi.2019.309.

Idrissov, A., Skec, S. and Maier, A.M. (2020), "Visualising Systems: Mapping System Features and Interactive Information Visualisations in Design", International Design Conference - DESIGN 2020.

Inselberg, A. (1985), “The plane with parallel coordinates”, The Visual Computer, Springer-Verlag, Vol. 1 No. 4, pp. 69-91, https://doi.org/10.1007/BF01898350.

Keim, D.A. (2002), "Information visualization and visual data mining", IEEE Transactions on Visualization and Computer Graphics, Vol. 8 No. 1, pp. 1-8, https://doi.org/10.1109/2945.981847.

Keller, R., Eger, T., Eckert, C.M. and Clarkson, P.J. (2005), "Visualising Change Propagation”, International Conference on Engineering Design (ICED’05), pp. 1-89.

Konyha, Z., Matković, K. and Hauser, H. (2009), Interactive Visual Analysis in Engineering: A Survey, Proc. Spring Conference on Computer Graphics (SCCG 2009).

Larkin, J.H., Simon, H.A. and Simon, A. (1987), "Why a Diagramm is ( Sometimes ) Worth Ten Thousand Words", Cognitive Science, Vol. 11, pp. 65-99, https://doi.org/10.1016/S0364-0213(87)80026-5.

Liang, J. and Huang, M.L. (2010), "Highlighting in information visualization: A survey", Proceedings of the International Conference on Information Visualisation, pp. 79-85, https://doi.org/10.1109/IV.2010.21.

Maier, A.M., Baltsen, N., Christoffersen, H. and Störrle, H. (2014), "Towards Diagram Understanding: A PilotStudy Measuring Cognitive Workload Through Eye-Tracking”, Proceedings of International Conference on Human Behaviour in Design 2014, No. October, pp. 1-6.

Munzner, T. (2014), Visualization Analysis \& Design, A K Peters/CRC Press, New York, USA, https://doi.org/10.1002/9781119978176.

Plaisant, C., Grosjean, J. and Bederson, B.B. (2002), "SpaceTree: Supporting exploration in large node link tree, design evolution and empirical evaluation”, IEEE Symposium on Information Visualization, pp. 57-64, https://doi.org/10.1109/INFVIS.2002.1173148.

Reingold, T. and Fruchterman, E. (1991), “Graph Drawing by Force-Directed Placement”, Software, Practice and Experience, Vol. 21 No. 11, pp. 1129-1164.

Scherr, M. (2008), "Multiple and Coordinated Views in Information Visualization”, Trends in Information Visualization, No. August, p. 38.

Shneiderman, B. (2003), “The Eyes Have It: A Task by Data Type Taxonomy for Information Visualizations", The Craft of Information Visualization, pp. 364-371, https://doi.org/10.1016/B978-155860915-0/50046-9.

Sim, S.K. and Duffy, A.H.B. (2003), "Towards an ontology of generic engineering design activities", Research in Engineering Design, Vol. 14 No. 4, pp. 200-223, https://doi.org/10.1007/s00163-003-0037-1.

Suwa, M. and Tversky, B. (1997), "What Do Architects and Students Perceive in their Design Sketches? A Protocol Analysis", Design Studies, Elsevier, Vol. 18 No. 4, pp. 385-403, http://dx.doi.org/10.1016/S0142694X(97)00008-2.

Ware, C. (2012), Information Visualization, Morgan Kaufmann.

Wehrend, S. and Lewis, C. (1990), "Problem-oriented Classification of Visualization Technique", IEEE Computer Society, pp. 139-143, 46, https://doi.org/10.1109/visual.1990.146375. 